\title{
Two Applications of Vectorial Color: Camera Design and Lighting of Colored Objects
}

\author{
James A. Worthey \\ 11 Rye Court, Gaithersburg, Maryland 20878-1901 \\ jim@jimworthey.com
}

\begin{abstract}
Color stimuli are described by 3-vectors, which can be added to model color mixing, yet vector diagrams are rare. A set of orthonormal opponent color matching functions leads to better vectors, solving old problems.

OCIS codes: (330.0330) Vision, color, and visual optics; (110.2970) Image detection systems.
\end{abstract}

\section{History of science and the peculiar status of lighting.}

Figure 1 reviews physics and engineering since about the time of Galileo, summarizing a longer chronology on the author's web site[1]. Most dates are from Wikipedia, but the longer timeline has other citations.

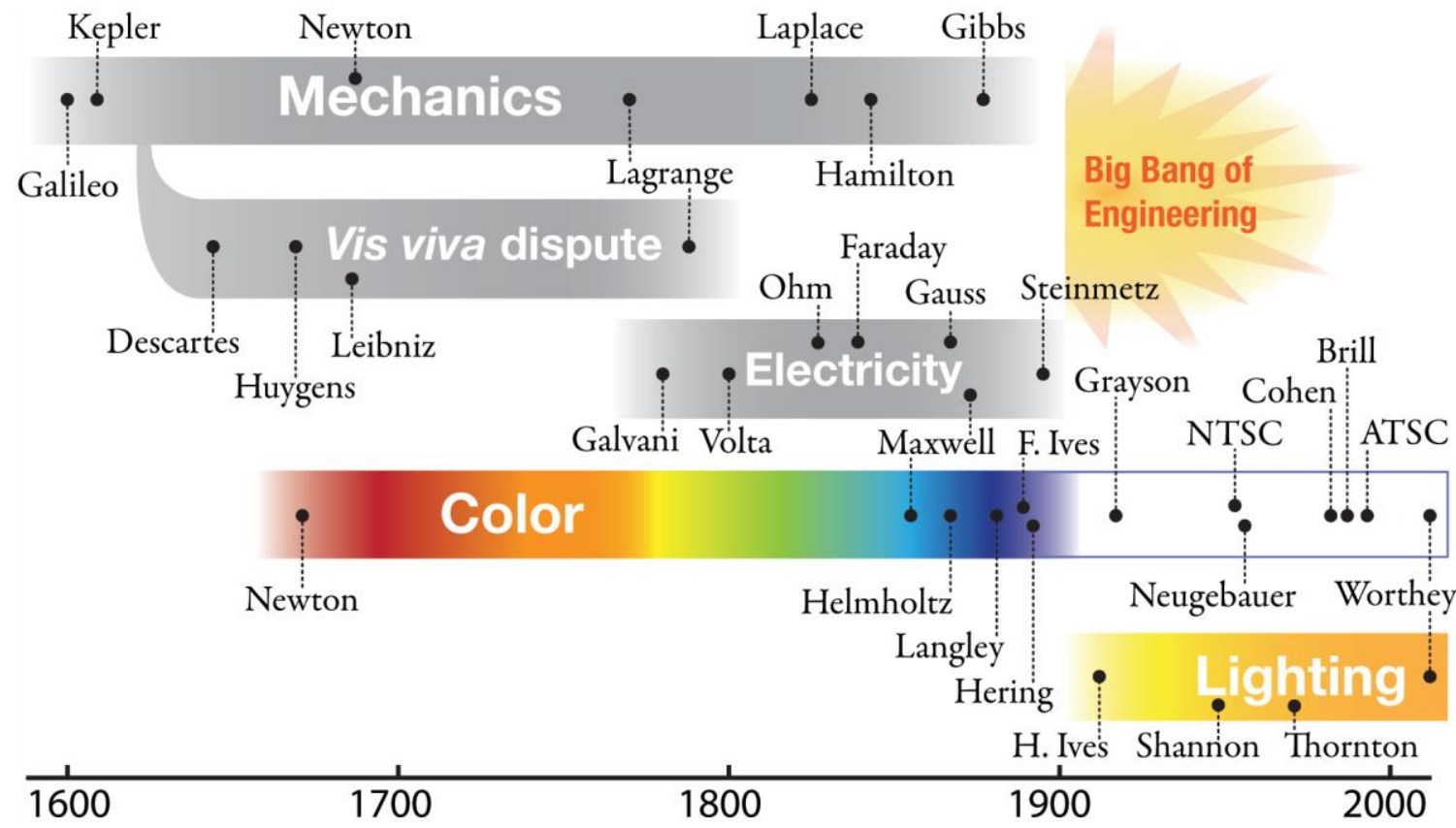

Fig. 1. Some key developments in physics since Galileo. Mechanics, meaning the study of motion, is a prototype for other developments. The topic called "Vis viva dispute" is based on Ref. [2]. Figure developed by Nicholas J. Worthey

Textbooks now teach that momentum $m v$ is conserved in a collision, but kinetic energy $1 / 2 m v^{2}$ can also be conserved. These confusing facts were resolved by the "vis viva dispute" [2], a topic in Fig. 1. In round numbers then, there was a 100-year age of vis viva within the 200-year era of mechanics. Systematic study of electricity took about 100 years. From Newton's "New Theory about Light and Colors" [3] to Frederic Ives's color photos based on the trichromatic theory of color vision[4] was about a 200-year era of color fundamentals.

In the 1800s, great minds worked to refine mathematical methods and complete classical physics. For example, Newton applied $F=m a$ to the motion of planets, but he did not have vector methods as we know them. Hamilton invented quaternions, which have important uses, but around 1900 Gibbs and others pieced together modern vector algebra.[5] Boltzmann and Gibbs advanced thermodynamics. Modern light measurements were launched by Langley's bolometer and Grayson's diffraction gratings.

\section{The big bang of engineering}

It was as if physicists were rushing to finish an old era and make way for the new. Then 1905 was Einstein's annus mirabilis, in which he published groundbreaking analyses of photoelectric effect, Brownian motion, relativity, and $E=m c^{2}$.[6] Physics turned toward new frontiers. In the larger world, Edison's first light bulb patent was granted 
in 1880 and in 1889 German-educated mathematician C. P. Steinmetz moved to the USA. The light bulb motivated a new electrical industry, where Steinmetz found work. Beyond his achievements as an inventor, he stands out as one who literally wrote the book for $20^{\text {th }}$ century electrical engineers. He invoked complex numbers to create so-called phasor methods for finding amplitude and phase of AC currents and voltages,[7] then published books on AC phenomena and other electrical subjects. As the gaps of classical physics were filled in, a transition occurred, shown in Fig. 1 as The Big Bang of Engineering. Authors such as Steinmetz found amongst great ideas a portion that could be immediately useful, creating workaday methods grounded in physics.

\section{The big fizzle of lighting}

Electrical engineering began with a great legacy, but lighting did not. For lighting, there is no Newton and no Steinmetz, even though Steinmetz himself wrote a book on the subject.[8] The book covers the physics of light and is still good reading. In the final chapter, he says the "engineering problem - to produce a definite light flux density throughout the illuminated space — can be solved... with any exactness." He then describes the gaps in knowledge: "Other physiological requirements are still very little understood or entirely unknown."[8]

Fig. 1 makes Claude Shannon a lighting expert, a bit of wishful thinking. He probably never thought about lighting, but gave birth to information theory in 1948.[9] Vision is an information channel, stimulated by contrasts, which usually arise from optical interaction between a light source and non-luminous objects. If we now step back to 1912, Herbert Ives tells us "It is, for instance, easily possible to make a subjective white, as by a mixture of monochromatic yellow and blue light. A white surface under this would look as it does under 'daylight' but hardly a single other color would."'[10] Fig. 2 shows what Ives had in mind. (H. Ives was OSA president, 1924-1925)

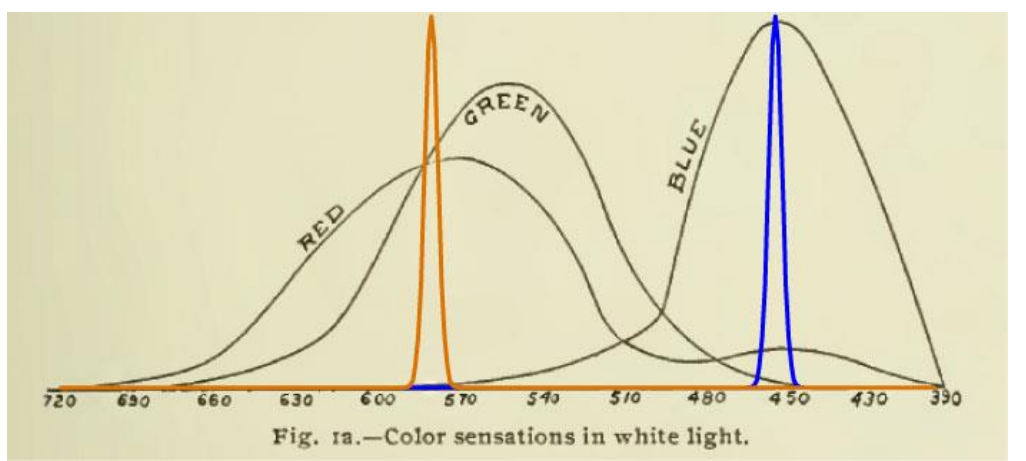

Fig. 2, a figure from H. E. Ives, Ref. [10]. The black lines are cone sensitivities from König. The colored curves were added by the present author as an example of the "mixture of monochromatic yellow and blue light" to which Ives refers.

The narrow bands are at 450 and $580 \mathrm{~nm}$. The "monochromatic" yellow stimulates both red and green receptors. Thus the two lights stimulate all 3 receptor systems, as daylight would do. Some 20 years before the Ives article, Ewald Hering had proposed that color vision is organized into blue versus yellow and red versus green. In those terms then, the two-bands light loses red-green contrasts among objects. One might say that colors are distorted, but Shannon - if he knew this example - would have recognized that the two-bands light diminishes information. Somewhat arbitrarily, we can say that this brief 1912 discussion marked the beginning of analytical thought about lighting. Ives combined basic knowledge of vision with simple optics, leading to a striking conclusion.

Since 1912, progress has been slow. The CIE's famous Color Rendering calculation,[11] in versions from 1965 to now does not mention simple examples or the loss of red-green contrasts. Thornton approached the same issue from a different direction, showing the benefits of a 3-bands light, set to certain wavelengths.[12] Bouma gave a cause-and-effect discussion of neodymium glass, which emphasizes red and green by absorbing some yellow.[13] During about 30 years I have developed analytical methods for describing the differences among light source spectra with emphasis on red-green contrast. Still, most discussions of lighting and color ignore Ives's simple insight from 100 years ago. We are still in the age of lighting, developing the algebra and words for a rationalized discussion.

\section{Vectorial Color}

So, Ives pointed out that two bands are bad, while Bouma and Thornton, each with his own examples, agreed that yellow is bad but red and green combined are good. Reasoning from evolution or experience might favor daylight. Fig. 3(a) is a modern version of Fig. 2, cone sensitivities, showing overlap of red and green. Those signals will be correlated, but color information can be separated out.[14] Fig. 3(b) shows the orthonormal opponent basis, a linear transformation of the cone functions. Specifically, $\mathrm{r}+\mathrm{g}$ with the right coefficients gives the achromatic function $\omega_{1}$, 
proportional to the usual y-bar. A red-green opponent function, $\omega_{2}$, is red minus green, orthogonal to $\omega_{1}$. Finally, $\omega_{3}$ is orthogonal to the others. Define $\Omega=\left[\omega_{1} \omega_{2} \omega_{3}\right]$, a matrix whose columns are the orthonormal set.[15] Basis $\Omega$ echoes Hering's intuitive idea of paired opposite colors. If $\mathrm{k}$ is the number of wavelength steps, maybe 31 or 471 , then $\Omega$ has dimensions $\mathrm{k} \times 3$. Orthonormality implies $\Omega^{\mathrm{T}} \Omega=\mathrm{I}_{3 \times 3}$, where $\mathrm{I}$ is an identity matrix and superscript $\mathrm{T}$ means transpose.
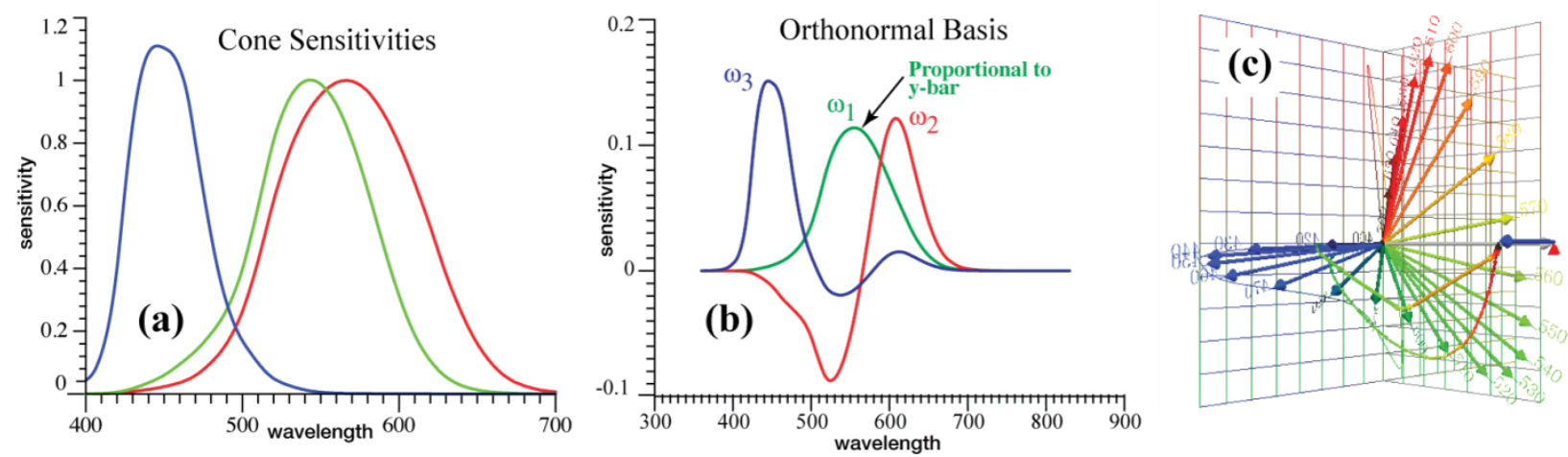

Fig. 3, (a) Human cone sensitivities; (b) The orthonormal basis $\Omega$; (c) The locus of unit monochromats, plus a lighting example.

Now recall Cohen's Matrix $R$, derived to be $R=A\left(A^{\mathrm{T}} A\right)^{-1} A^{\mathrm{T}}$. The columns of matrix $A_{\mathrm{k} \times 3}$ are the cone sensitivities, or any reversible linear transformation of them. The original goal was to take any power spectrum $L(\lambda)$ and find its projection into the vector space of $A$, called $L^{*}$, the fundamental metamer of $L$. The * is Cohen's notation, and has nothing to do with complex conjugate; it means projection. Then $L^{*}=R L$. Used thus, $R$ performs a curve fit, equivalent to using a pseudoinverse, but matrix $R$ has important properties. If $A$ is replaced by any linear transformation of itself, $R$ is invariant in the strongest sense; it is the same large array of numbers (e.g. $471 \times 471$ ). The columns (or rows) of $R$ are the fundamental metamers of the spectrum, which trace the Locus of Unit Monochromats (LUM). One linear transformation of $A$ is $\Omega$, which leads to $R=\Omega \Omega^{\mathrm{T}}$. But recall, $\Omega^{\mathrm{T}} \Omega=\mathrm{I}_{3 \times 3}$.

In Fig. 3(c), the arrows from the origin trace points along the LUM, a curve that's invariant because $R$ is. Strangely but simply enough, the rows of $\Omega$ trace the LUM in the specific coordinate system, in which the axes are white, red-green, and blue-yellow. A white light maps to a vector from the origin, and if one breaks the spectrum into 10-nm bands, the vectors can be added band-by-band to the same white. In the figure the arcing vector chain is daylight, while the chain for a matching mercury vapor light takes a shortcut to the same white point. That shows the matching-but-different quality as in H. Ives's example, in a detailed way open to further analysis.

Following Maxwell, Frederic Ives (father of Herbert) observed that camera sensors should mimic the eye.[4] In a modern statement, the camera sensors should be a linear transformation of cone sensitivities. By the invariance of the LUM, a camera's LUM should resemble that of the eye. The Fit First Method, based on a few matrix operations, allows a detailed comparison of camera and eye, directly using Cohen's discovery of the invariant LUM.[16]

\section{References}

[1] http://www.jimworthey.com .

[2] George E. Smith, "The vis viva dispute: A controversy at the dawn of dynamics," Physics Today, October 2006, pp. 31-36.

[3] Isaac Newton, "New Theory about Light and Colors," Phil. Trans. 6:2075-3087, 1671.

[4] Frederic Eugene Ives, A new principle in heliochromy. Philadelphia: Printed by the author; 1889.

[5] Michael J. Crowe, A history of vector analysis, (Dover Press, New York, 1985).

[6] Wikipedia article: Annus mirabilis papers.

[7] Charles P. Steinmetz, Theory and calculation of alternating current phenomena, $7^{\text {th }}$ Ed., New York: McGraw-Hill, 1916. On the web.

[8] Charles P. Steinmetz, Radiation, Light and Illumination, New York: McGraw-Hill, 1909. On the web.

[9] Claude E. Shannon, "A Mathematical Theory of Communication," Bell System Technical Journal, 27:379-423, 623-656, 1948. On the web.

[10] Herbert E. Ives, "The relation between the color of the illuminant and the color of the illuminated object," Transactions of the Illuminating

Engineering Society 7:62-72.(1912). On the web.

[11] http://en.wikipedia.org/wiki/Color rendering index

[12] William A. Thornton, "Luminosity and color-rendering capability of white light," J. Opt. Soc. Am. 61(9):1155-1163 (September 1971).

[13] P. J. Bouma, "The colour reproduction of incandescent lamps and 'Philiphane Glass,"” Philips Technical Review 3:47-49 (1938).

[14] Gershon Buchsbaum and A. Gottschalk, "Trichromacy, opponent colours coding and optimum colour information transmission in the retina," Proc. R. Soc. Lond. B 220, 89-113 (1983).

[15] James A. Worthey, "Vectorial color," Color Research and Application, 37(6):394-409 (December 2012).

[16] James A. Worthey, "Applications of vectorial color," Color Research and Application, 37(6):410-423 (December 2012). 


\title{
Two Applications of Vectorial Color: Camera Design and Lighting of Colored Objects
}

\author{
James A. Worthey \\ 11 Rye Court, Gaithersburg, Maryland 20878-1901 \\ jim@jimworthey.com

\section{Erratum:}

In the middle of the second-to-last paragraph, the following sentence appears: "A white light maps to a vector from the origin, and if one breaks the spectrum into 10-nm bands, the vectors can be added band-by-band to the same white."

The sentence may be easier to understand with added word or two: "A white light maps to a vector from the origin, and if one breaks the spectrum into 10-nm bands, the vectors can be added band-by-band to yield the same white."

In other words, for each $10 \mathrm{~nm}$ band there is a vector, and if you add the vectors (vectorially!) then you get the same white as if you would calculate the vector some other way. While you could sum vectors in the XYZ system, the whole exercise makes more sense in the invariant color space, which is the intention here. 